\title{
La dificultad en la permanencia de estudiantes indígenas en la carrera Enseñanza del Inglés, Campus Coto
}

\section{Difficulties for indigenous students to remain in the English Teaching major in Campus Coto}

\author{
Dafne Atencio García \\ Universidad Nacional \\ Sede Regional Brunca, Campus Coto \\ Puntarenas, Costa Rica \\ dafneatencio@gmail.com \\ María Gómez Rojas \\ Universidad Nacional \\ Sede Regional Brunca, Campus Coto \\ Puntarenas, Costa Rica \\ mariagomez98.mg@gmail.com
}

\section{Recibido: 21/04/18 Aceptado: 31/07/18}

Resumen. Este estudio muestra una situación que se presentó en los estudiantes pertenecientes a la población indígena de la carrera de Enseñanza del Inglés del Campus Coto. Dichos estudiantes lograron acceder a la educación universitaria, sin embargo, esta población, de acuerdo a las entrevistas realizadas y a lo observado estadísticamente en los años anteriores, no logra permanecer en la carrera. En algunos casos no logran pasar el primer curso que se recibe de la lengua inglesa (en este caso Inglés Integrado 1), como consecuencia, los estudiantes deciden desertar temporal o definitivamente. De igual manera, el porcentaje de alumnos que logran concluir con la carrera es bajo. Debido a los hechos anteriores, esta investigación tiene como objetivo conocer cuál es el factor más significativo que causa que los estudiantes de población indígena no logren terminar, deserten o repitan continuamente cursos de la carrera de Enseñanza del Inglés. Estos discentes además de tener que sobrepasar el desafío de alejarse de sus territorios, deben enfrentar un choque cultural entre su lengua indígena, la lengua castellana y la lengua a adquirir, en este caso, el inglés. Asimismo, se investigaron los registros acerca de cuántos estudiantes eran admitidos en la carrera mencionada y el posible cambio de los mismos para otras carreras. Siendo así, la carrera de Enseñanza del Inglés es un objetivo desafiante para la población indígena de la zona.

Palabras clave: Campus Coto, choque cultural, inglés, estudiantes, indígenas. 


\begin{abstract}
This study shows a situation presented to students from the indigenous population of the English teaching major of Campus Coto. These students managed to access university education; however, according to the interviews conducted and what was observed statistically in previous years, this population is not able to go successfully through the major. In some cases, they fail to pass the first course that is received from the English language (in this case integrated English 1); as a result, students decide to defect temporarily or permanently. Similarly, the percentage of students who manage to conclude with the major is low. Due to the previous facts, this research aimed to find out what the most significant factor was, which causes students of the indigenous population not to finish, drop or continuously repeat courses of the English teaching major. Besides having to overcome the challenge of moving away from their territories, these students face a cultural clash between their indigenous language, Spanish, and the language to acquire, in this case, English. Likewise, records were analyzed to know about how many students were admitted to the major mentioned before, as well as the possible changes of students to other majors were examined. In this case, the English teaching major has been transformed into one of the most challenging objectives for the indigenous population of the area.
\end{abstract}

Keywords: Campus Coto, cultural Clash, English,students, indigenous.

La presente investigación consiste en la evaluación de un factor que influye tanto en el interés social como en lo personal de los estudiantes indígenas matriculados en la carrera de Enseñanza del Inglés del Campus Coto. Además de la preocupación social, se quiere explorar el motivo que impide que estos estudiantes puedan continuar en la carrera. Conociendo el factor que está causando este problema se pretende tener la opción de ayudar o aconsejar sobre posibles soluciones a los entes administrativos del Campus Coto y así lograr que esta población termine efectivamente con sus estudios en el área del inglés en el tiempo determinado para esto.

Uno de los factores a considerar es el nivel del idioma español que los estudiantes indígenas tienen al ingresar a la carrera, debido a que las primeras clases de Inglés se relacionan con el idioma español, y el estudiante debe crear lo que se conoce como aprendizaje significativo, que es la relación entre lo que ya es considerado como aprendido y el nuevo conocimiento que se está adquiriendo. Por lo tanto, el desconocimiento, tanto de los estudiantes del idioma español como de los profesores del idioma indígena, genera no solo dificultad al comunicarse, sino también un choque cultural entre tres diferentes culturas (los idiomas español e inglés y el idioma nativo que los estudiantes posean). 
URL: http://www.revistas.una.ac.cr/index.php/dialogo/index

CORREO ELECTRÓNICO: universidadendialogo@una.cr

DOI: https://doi.org/10.15359/udre.9-1.3

Debido a esto, se busca identificar por medio de encuestas y diferentes fuentes de información las dificultades que impiden que los estudiantes indígenas de Enseñanza del Inglés del Campus Coto logren terminar satisfactoriamente la carrera, para un conocimiento propio y para futuras investigaciones a realizarse. De igual manera, se busca facilitar a los estudiantes herramientas que les permitan la superación de esta barrera cultural y demás desventajas a superar.

En materia educativa, los pueblos indígenas han cuestionado los esquemas integracionistas que han privado en la currícula, así como el proceso de exclusión que opera en el sistema educativo, debido a factores como el disminuido acceso a la información, la condición socioeconómica tan limitada de estas poblaciones y la discriminación cultural, entre otros. (Alfaro, Arias, Cerdas, Hernández, Pino, Segura y Zúñiga, 2013, p. 8)

La igualdad de oportunidades en relación con la educación entre las distintas poblaciones de Costa Rica es un dilema que siempre se ha buscado resolver. Rojas (2002) menciona que nuestro país al contar con una gran cantidad de población indígena busca brindar un nivel superior de educación para esta población, que muchas veces es discriminada en cuanto a las oportunidades educativas. Además, se tomó en consideración por qué parece ser común la puesta en decisión de desertar de parte de estos estudiantes.

En el país se han creado planes para el soporte de esta población a nivel educativo; tal es el caso del Plan Quincenal: "El PPIQ tiene como propósito, el mejoramiento en las condiciones de acceso y permanencia de estudiantes indígenas en la educación superior pública, con especial atención, a la pertinencia cultural de las acciones que las instituciones realicen con esos propósitos" (Alfaro et al., 2013, p. 11).

\section{Desarrollo}

La Universidad Nacional de Costa Rica en su rol educativo es una institución no solo humanista, sino también inclusiva. Como prueba de ello en cada una de sus sedes y campus se presentan diferentes actividades y programas para la población en general. Campus Coto no es la excepción, en este se imparten cuatro carreras integradas cada una con aproximadamente treinta estudiantes o más. La Universidad Nacional ofrece un variable número de cupos para que estudiantes de territorios indígenas sean recibidos sin examen de admisión. Esto brinda un alto nivel de educación para aquellos que la quieran y no tengan los medios para acceder a ella. 
Revista UniversidAd en DiÁlogo • Vol. 9, N. ำ 1, Enero-Junio, 2019 • 47-62

ISSN 2215-2849 • EISSN: 2215-4752

URL: http://www.revistas.una.ac.cr/index.php/dialogo/index CORREO ELECTRÓNICO: universidadendialogo@una.cr

DOI: https://doi.org/10.15359/udre.9-1.3

Como lo muestra a continuación una de las noticias de la página de la Universidad Nacional: el ingreso de este grupo de jóvenes indígenas se enmarca en un sistema de admisión integral establecido por la UNA, en 2009, el cual garantiza las condiciones necesarias para que estudiantes de sectores vulnerables ingresen a la institución, permanezcan y culminen de manera exitosa su carrera. En el caso de la población indígena, el ingreso se produce mediante dos vías: la admisión regular y los programas de acción afirmativa, diseñados para compensar la situación de desventaja que acumulan los pueblos originarios, mediante un tratamiento preferencial. Es así como, al 2017, un total de 395 estudiantes de primer ingreso provenientes de colegios ubicados en territorios indígenas en zonas como Talamanca, Matina, San Vito, Térraba, Brujo, Corredores, Guatuso y Buenos Aires, se han matriculado en la institución, de los cuales ochenta han ingresado por el sistema de admisión tradicional y 315 gracias a programas de acción afirmativa.

Estos veintidós indígenas que tendrán acceso a la educación superior, a partir de 2018, forman parte de las zonas vulnerables a cuyas necesidades ha dado respuesta la UNA, mediante lo que la rectora adjunta, Luz Emilia Flores, califica en el periódico Campus Digital de la Oficina de Comunicación de la Universidad Nacional como “...una educación inclusiva, equitativa, más comprometida con nuestra Madre Tierra y más sensible a las necesidades de las poblaciones" (julio, 2017).

Los estudiantes indígenas que ingresan a la educación superior gracias al Programa de Salvaguarda Indígena no pueden cambiarse de carrera a menos que realicen el examen de admisión del año siguiente y logren entrar en la carrera del mismo modo que los demás aspirantes. Lamentablemente, no existe un documento en el cual se puedan identificar los objetivos ni información relevante para el Programa Salvaguarda Indígena, debido a que este es un acuerdo que se realiza entre los miembros del Consejo de Administración Extraordinaria. Este consejo es el encargado de determinar cuántos cupos se abren por carrera para los estudiantes indígenas que quieran ingresar al campus por medio del Programa Salvaguarda Indígena.

Alfaro et al. (2013) mencionan en el Plan Quincenal que la Universidad Nacional realizará anualmente una valoración de cupos en carreras que se ofrecen en las Sedes Regionales, para ofrecer por lo menos cinco cupos en cada una de ellas a jóvenes indígenas que tengan el bachillerato aprobado. Este estudio incluirá valorar si se solicita o no la prueba de admisión. 
URL: http://www.revistas.una.ac.cr/index.php/dialogo/index

CORREO ELECTRÓNICO: universidadendialogo@una.cr

DOI: https://doi.org/10.15359/udre.9-1.3

Cabe destacar que esta población recibe un soporte extra por parte de la Universidad, sin embargo, no logran avanzar en la carrera de Enseñanza del Inglés. Entre la ayuda brindada por parte de la Universidad están las becas, los cursos de inducción y el seguimiento de vida universitaria y residencia universitaria.

El Programa Salvaguarda Indígena fue instituido hasta el año 2014 en el Campus Coto, pero entró a regir hasta el año 2015, como lo muestran los siguientes datos facilitados por la directora de Bienestar Estudiantil del Campus Coto, Mayra Segura García.

Tabla 1

Estudiantes ingresados anualmente por medio del Programa Salvaguarda desde su gestión en el Campus Coto

\begin{tabular}{lll}
\hline Año & Número de ingresos & Número de estudiantes activos en la carrera \\
\hline $\mathbf{2 0 1 5}$ & 2 & 1 \\
$\mathbf{2 0 1 6}$ & 3 & 1 \\
$\mathbf{2 0 1 7}$ & 3 & 2 \\
\hline Total: & 8 & 4 \\
\hline
\end{tabular}

Nota: Datos facilitados por la directora de Bienestar Estudiantil del Campus Coto, Mayra Segura García

A pesar de que no existe un documento que especifique los detalles del programa, se mantiene un registro en vida estudiantil, el cual se ve reflejado en la tabla anterior.

De los tres estudiantes que se matricularon en la institución en el año 2017, solo continúan en la Universidad dos. Ninguno de ellos consiguió concluir el primer semestre de la carrera satisfactoriamente. Dichos estudiantes reprobaron el primer curso de lengua inglesa (Inglés Integrado 1). Estos estudiantes se encuentran activos en la Universidad, recibiendo otros cursos de la carrera, como los pedagógicos y generales. Además, reciben tutorías con el resto del grupo de Enseñanza del Inglés de primer ingreso. 


\section{Metodología}

Los participantes elegidos para esta investigación fueron los estudiantes indígenas que se encontraban o estuvieron matriculados en la carrera de Enseñanza del Inglés de la Universidad Nacional en el Campus Coto. La cantidad de estudiantes entrevistados fue la siguiente: dos estudiantes de primer ingreso y dos estudiantes que ingresaron a la carrera en los años anteriores; de estos, dos continúan activos en la carrera de Enseñanza del Inglés; uno en la carrera de Administración; y el último continúa en la Universidad, pero no de forma activa, ya que reprobó el curso por segunda vez consecutiva. Cabe agregar que las personas entrevistadas fueron tres hombres y una mujer.

El Campus Coto cuenta con diez profesores de Enseñanza del Inglés, los profesores entrevistados fueron cinco, debido a que ellos han sido los encargados de impartir las lecciones de Inglés Integrado 1 en los últimos años.

\section{Instrumento}

Para la recolección de datos se utilizó una encuesta. Dicho instrumento incluía trece preguntas, entre ellas preguntas abiertas y cerradas. El objetivo de esta fue conocer la perspectiva de los estudiantes que se ven envueltos en esta situación e identificar los posibles factores que afectan su efectivo progreso en la carrera de Enseñanza del Inglés. Adicionalmente, se les realizó un cuestionario para determinar el nivel de español con el cual esta población ingresa a la educación superior. Esto con el objetivo de determinar si el nivel de español adquirido afecta su proceso de aprendizaje de la nueva lengua. Además de la realización de cuestionarios a los estudiantes, se realizó una pequeña encuesta de cinco preguntas abiertas a los profesores encargados de impartir lecciones a primer ingreso. Esta misma encuesta se realizó a la tutora responsable de brindar tutorías a dichos estudiantes en el año 2017. Esto con el objetivo de conocer sus perspectivas y opiniones acerca del caso bajo investigación.

\section{Resultados}

Los profesores concuerdan con que los estudiantes traen consigo una desventaja académica desde el colegio, por lo tanto, al ingresar a la universidad se encuentran en etapas de transición y adaptación que requieren un mayor acompañamiento. Los profesores indican que estos estudiantes participan muy poco y que necesitan una inducción tanto antes de ingresar al proceso universitario como durante. Esta inducción debe ser prolongada para solventar 
URL: http://www.revistas.una.ac.cr/index.php/dialogo/index

CORREO ELECTRÓNICO: universidadendialogo@una.cr

DOI: https://doi.org/10.15359/udre.9-1.3

las desventajas que los estudiantes traen consigo desde el colegio. Debido a que en dos o tres lecciones es difícil equilibrar a los estudiantes.

Los profesores mencionan que algunos factores que podrían estar afectando a esta población, a parte del poco conocimiento de la lengua a estudiar, son el desconocimiento en el uso de tecnología, la falta de cohesión grupal, el poco aprovechamiento de horas atención, el conformismo por condición socioeconómica, factores personales, el desconocimiento de estrategias de estudio, la falta de exposición a lenguas foráneas (español e inglés), la falta de integración en el grupo de estudio, la influencia de algunas prácticas de la cultura madre. Aspectos relacionados con la personalidad. Además ellos mencionan que la parte cultural del idioma afecta a estos estudiantes. Se cree que el ser humano para aprender otro idioma se aferra al idioma oriundo.

Asimismo, un factor preocupante es la posibilidad de que los estudiantes no posean un buen español, por lo que profesores del campus indican algunas iniciativas que se podrían tomar en consideración para aumentar la permanencia y asegurar el avance de estos estudiantes en la carrera. Ellos citan iniciativas como un curso introductorio intensivo, proveer un tutor para el grupo de Salvaguarda Indígena durante el primer año, una mejora en la nivelación de los estudiantes, debido a que esta debe darse de manera gratuita desde que están en el colegio. Además, mejorar la malla en función de todos los estudiantes, no solo para esta población. Usar una metodología de best friend o mentor para crear así una mejor relación y aculturación.

Uno de los puntos a los que hacen mención es que no solo esa carrera, sino todas las demás son aptas para todo tipo de estudiante, siempre y cuando exista máximo compromiso por parte de los participantes en el proceso educativo. Estos estudiantes poseen una desventaja, mas no una limitación.

\section{Resultados de la encuesta de estudiantes}

La encuesta realizada al estudiante repitente dio los siguientes resultados: el estudiante considera la carrera de Enseñanza del Inglés difícil, mencionó que no había recibido inglés anteriormente, ni en la escuela ni en el colegio. Considera que el ambiente en las clases es de una comodidad regular y opina que su relación con los compañeros es buena. Con respecto a lo académico, menciona que dedicaba tres horas por semana al estudio, además de que evacuaba las dudas por medio de los profesores y las tutorías. Él considera que los profesores del campus sí explican bien, pero entendería mejor las clases si 
Revista UniversidAd en DiÁlogo • Vol. 9, N. ำ 1, Enero-Junio, 2019 • 47-62

ISSN 2215-2849 • EISSN: 2215-4752

URL: http://www.revistas.una.ac.cr/index.php/dialogo/index CORREO ELECTRÓNICO: universidadendialogo@una.cr

DOI: https://doi.org/10.15359/udre.9-1.3

estas se impartieran en el idioma español. El estudiante señala que escogió la carrera de Enseñanza del Inglés porque le gustaba y trató de esforzarse lo más que pudo, sin embargo, es muy difícil debido a que el problema empieza desde el colegio, debido a la mala formación en la materia y cuando se ingresa a la universidad el contenido del curso presenta un nivel muy alto en comparación con el inglés del colegio. Pensó que empezaría con un nivel básico, pequeños detalles hasta desarrollar un nivel alto de literatura y lenguaje.

También menciona que la promoción de la carrera se hizo por medio del Programa Salvaguarda Indígena, donde les indicaron el día, la fecha y la hora de la charla. Luego les indicaron cómo ingresar al proceso. Él indica que las tutorías son un espacio en el cual se aprende mucho, sin embargo, no son tan efectivas porque muchas veces el tutor desarrolla los temas de una manera diferente al profesor, lo cual crea inseguridad en el estudiante.

\section{Estudiantes activos en la carrera}

Existen ciertas diferencias entre las respuestas de los dos estudiantes. Uno menciona no haber recibido inglés anteriormente y el otro sí. Uno de ellos percibió la carrera difícil y el otro fácil. Ambos se sienten cómodos en el ambiente de clase y se relacionan con más de cinco compañeros. Uno de los estudiantes dijo haber recibido la información por medio de visitas de la universidad y el otro menciona que no recibió nada de información relevante sobre el proceso. Ambos mencionan que escogieron la carrera porque les gusta y porque les servirá para el futuro. En relación con lo académico, ambos mencionan que los profesores hacen su trabajo de la mejor manera y no concuerdan con el factor de que si se impartieran las clases en español sería más fácil. Uno de los estudiantes mencionó que por semana estudia siete horas o más; al contrario, el otro estudia pocas horas (de una a tres horas). Ellos mencionan que evacuan las dudas por medio del profesor y de los compañeros o por medio de tutorías, ya que ambos asisten a ellas una vez por semana. Por último, uno de los estudiantes que perteneció a la carrera, pero hizo el cambio, contestó a la misma encuesta en la cual señaló que el nivel de la carrera era difícil y que la relación con los compañeros y profesores era muy amigable. En caso de que el estudiante necesitara algo, los compañeros y profesores de la misma materia estaban siempre anuentes a contestar y resolver sus dudas. Un aspecto interesante que 
URL: http://www.revistas.una.ac.cr/index.php/dialogo/index

CORREO ELECTRÓNICO: universidadendialogo@una.cr

DOI: https://doi.org/10.15359/udre.9-1.3

destaco es que, aunque se dice que hay promoción de las carreras del Campus Coto, en este punto el estudiante respondió a la pregunta con un no. Además, considera que si los profesores de los cursos en Inglés añadieran el idioma español más frecuentemente sería más fácil entender el nuevo idioma.

La tutora menciona que en las tutorías estos estudiantes tratan de evacuar la mayor cantidad de dudas posibles y que participan bastante. Ella mencionó que muchas veces es difícil establecer un equilibrio entre el conocimiento de la lengua y los nuevos contenidos a desarrollarse. También señala que un factor importante que podría determinar el éxito de estos estudiantes es el apoyo por parte del profesor.

\section{Conclusión}

La investigación realizada muestra que los estudiantes de población indígena, ingresados por medio del Programa de Salvaguarda Indígena, muestran distintos factores que impiden el avance provechoso en la carrera de Enseñanza del Inglés. A pesar de que los estudiantes, de acuerdo a las encuestas tomadas de la página web Cervantes (página web que sirve para conocer el nivel de español que se tiene), obtuvieron un nivel $\mathrm{C} 1$ en el idioma español, los profesores mencionan que este es un gran factor que muchas veces limita el desenvolvimiento de los individuos, debido a que no en todas las residencias de estos, ya sean reservas o pueblos aledaños, reciben la educación en idioma español. Entre los estudiantes se destaca el factor de no recibir una adecuada preparación en el idioma foráneo en los colegios, y debido a esto los estudiantes presentan una desventaja que incluso con un pequeño curso de inducción es difícil de solventar. Algo importante a destacar es que sí existe un choque cultural en los estudiantes al ingresar al sistema universitario. Debido a la necesidad de usar el idioma español con más frecuencia, los estudiantes pasan por un proceso de adaptación tanto con el nuevo idioma como con la nueva cultura.

Se puede concluir que esta dificultad de los estudiantes se inclina hacia un no conocimiento profundo de la lengua, pero también al proceso de adaptación. Para solventar la desventaja académica se proponen constantes talleres de estrategias de aprendizaje y más horas de tutorías para esta población. Además, un acompañamiento del estudiante durante el proceso universitario basado en los medios de aprendizaje del lenguaje. 


\section{Referencias bibliográficas}

Alfaro, F., Arias, J., Cerdas, Y., Hernández, E., Pino, G., Segura, D. y Zúñiga, X. (2013). Plan para pueblos indígenas (PPIQ). Recuperado de http://www.documentos.una.ac.cr/bitstream/handle/unadocs/3291/ Plan\%20Quinquena 1\%20para\%20Pueblos\%20Ind\%C3\%ADgenas\%20Quinquenal\%20e\%20Interuniversit ario\%20\%28PPIQ\%29. pdf? sequence $=1 \&$ isAllowed $=y$

Oficina de Comunicación de la Universidad Nacional (julio, 2017). Una graduación inclusiva y diversa. Campus Digital. Recuperado de http:// www.campus.una.ac.cr/2017julio_pag21.html

Rojas, C. (S. f.). La enseñanza de las lenguas indígenas en Costa Rica. Recuperado de http://www.revistas.una.ac.cr/index.php/EDUCARE/article/ viewFile/997/921

Test Your Spanish. Recuperado de http://www.cervantes.to/test_inicial.html

Universidad Nacional de Costa Rica (01 de junio, 2017). Indígenas optan por la UNA. Hoy en el Campus. Recuperado de http://www.hoyenelcampus.una.ac.cr//index2.php?option=com_content\&do_pdf=1\&i d=2186 
URL: http://www.revistas.una.ac.cr/index.php/dialogo/index

CORREO ELECTRÓNICO: universidadendialogo@una.cr

DOI: https://doi.org/10.15359/udre.9-1.3

\section{Anexos}

Universidad Nacional

Campus Coto

Estudiantes: Dafne Atencio García y María Gómez Rojas

\section{Encuesta para estudiantes}

Objetivo: El objetivo de esta encuesta es conocer la opinión de los estudiantes indígenas de la carrera Enseñanza del Inglés, con la finalidad de conducir la respectiva investigación. La información suministrada será utilizada para propósitos académicos únicamente.

Instrucciones: Lea cuidadosamente y conteste las siguientes preguntas de acuerdo con lo que se le solicita.

1. ¿Cómo percibe usted la carrera?
a) Difícil
b) Regular
c) Mal

2. ¿Recibió Inglés anteriormente?
a) Sí
b) No
c) Escuela / Colegio

3. ¿Cuánta comodidad tiene en los cursos?
a) Excelente
b) Buena
c) Regular
d) Mala

4. ¿Cómo considera su relación con sus compañeros?
a) Excelente
b) Buena
c) Regular
d) Mala

5. ¿Con cuántos compañeros se comunica diariamente?
a) Más de uno
b) Más de tres
c) Más de cinco
d) Ninguno 
Revista Universidad en DiÁlogo • Vol. 9, N. ำ 1, Enero-Junio, 2019 • 47-62

ISSN 2215-2849 • EISSN: 2215-4752

URL: http://www.revistas.una.ac.cr/index.php/dialogo/index CORREO ELECTRÓNICO: universidadendialogo@una.cr

DOI: https://doi.org/10.15359/udre.9-1.3

6. ¿Por qué escogió esta carrera?

7. ¿Cuál fue su primera impresión de la carrera?

8. ¿Cómo fue la promoción de la carrera en su colegio, comunidad o territorio?
a) Charlas
b) Visitas de la universidad
c) No recibió ninguna información
d) Otro. Especifique

9. ¿Considera usted que los profesores de Inglés explican de la mejor manera los contenidos del curso?

$$
\text { Sí ( ) No ( ) }
$$

10. ¿Cuántas horas de estudio dedica por semana?
a) De una a tres horas
b) De tres a siete horas
c) Siete horas o más

11. ¿Cree usted que si el profesor usara el idioma español en clases entendería mejor los contenidos?

$$
\text { Sí ( ) No ( ) }
$$

12. ¿Cuando usted no entiende algún tema solicita ayuda? ¿A quién?

13. ¿Asiste usted a las horas tutoría? Justifique su respuesta.

$$
\text { Sí ( ) No ( ) }
$$


URL: http://www.revistas.una.ac.cr/index.php/dialogo/index

CORREO ELECTRÓNICO: universidadendialogo@una.cr

DOI: https://doi.org/10.15359/udre.9-1.3

\section{Encuesta de nivel del idioma español Nivel de español C1}

1. __ bun chico muy abierto. Le encanta hablar con todo el mundo.

a) Es

b) Esta

2. Se me las llaves.
a) He perdido
b) Han perdido
c) Ha perdido

3. No creo que lo
a) Había hecho
b) Hubiera hecho
c) Hizo

4. La mujer vimos ayer, es la mujer de Juan.
a) Quien
b) Que

5. Sentí que alguien a la puerta.
a) Llamara
b) Llamaba

6. Viene porque le obligan y no porque
a) Quiere
b) Quiera

7. Ojalá___al concierto, pero estuve enfermo.
a) Fuera
b) Hubiera ido
c) Vaya

8. Me di cuenta de que había olvidado las llaves llegar a la oficina.
a) Nada más
b) Cuando
c) Tan pronto como 
Revista UniversidAd en DiÁlogo • Vol. 9, N. ำ 1, Enero-Junio, 2019 • 47-62

ISSN 2215-2849 • EISSN: 2215-4752

URL: http://www.revistas.una.ac.cr/index.php/dialogo/index CORREO ELECTRÓNICO: universidadendialogo@una.cr DOI: https://doi.org/10.15359/udre.9-1.3

9. Date prisa que los invitados llegar.
a) Están a punto de
b) Se ponen a
c) Vienen a

10. No me gusta este piso: es pequeño, frío y, , caro.
a) Además
b) Entonces
c) Igual 
URL: http://www.revistas.una.ac.cr/index.php/dialogo/index

CORREO ELECTRÓNICO: universidadendialogo@una.cr

DOI: https://doi.org/10.15359/udre.9-1.3

Universidad Nacional

Campus Coto

Estudiantes: Dafne Atencio García y María Gómez Rojas

\section{Encuesta para profesores de la carrera}

Objetivo: El objetivo de esta encuesta es conocer la perspectiva de los profesores encargados de la enseñanza del idioma inglés, con referencia a la situación educativa de la población indígena del Campus Coto. La información suministrada será utilizada para propósitos académicos únicamente.

Instrucciones: Lea cuidadosamente y conteste las siguientes preguntas de acuerdo con lo que se le solicita.

1. ¿Cómo considera el desenvolvimiento de los estudiantes salvaguarda durante su clase? Justifique su respuesta.

( ) Excelente
( ) Bueno
( ) Malo

2. ¿Considera usted que los estudiantes inscritos en la carrera por medio del Programa Salvaguarda Indígena necesitan recibir un curso para equilibrar y compensar el nivel necesario antes de recibir Integrado 1? Justifique su respuesta.
( ) Sí
( ) No

3. ¿Cuáles son algunos factores que podrían estar afectando el rendimiento académico del estudiante salvaguarda? 
Revista UniversidAd en DiÁlogo • Vol. 9, N. ำ, Enero-Junio, 2019 • 47-62

ISSN 2215-2849 • EISSN: 2215-4752

URL: http://www.revistas.una.ac.cr/index.php/dialogo/index CORREO ELECTRÓNICO: universidadendialogo@una.cr

DOI: https://doi.org/10.15359/udre.9-1.3

4. De acuerdo con su opinión. ¿Cuáles aspectos de la carrera se podrían fortalecer para una mejor promoción de los estudiantes salvaguarda? Explique.

5. ¿Considera usted que la carrera Enseñanza del Inglés es una buena opción para los estudiantes salvaguarda? Justifique su respuesta.

Gracias por su colaboración 